\title{
Comparison of ultracentrifugation and density gradient separation methods for isolating Tca8113 human tongue cancer cell line-derived exosomes
}

\author{
ZHUOYUAN ZHANG ${ }^{1,2}$, CHENXING WANG $^{1,2}$, TANG LI $^{1,2}$, ZHE LIU $^{1,2}$ and LONGJIANG LI ${ }^{1,2}$ \\ ${ }^{1}$ State Key Laboratory of Oral Diseases and ${ }^{2}$ Department of Head and Neck Cancer Surgery, \\ West China School of Stomatology, Sichuan University, Chengdu, \\ Sichuan 610041, P.R. China
}

Received September 14, 2013; Accepted March 28, 2014

DOI: $10.3892 / \mathrm{ol} .2014 .2373$

\begin{abstract}
The aim of the present study was to compare the method of ultracentrifugation and density gradient separation for isolating Tca8113 human tongue squamous cell carcinoma cell line-derived exosomes. The exosomes were obtained from the culture supernatant of cultured Tca8113 cells, respectively, followed by identification with transmission electron microscopy observation and western blot analysis. The two different methods were then compared by the morphology, the distribution range of the particle size and the concentration of proteins of the extracted exosomes. In vitro, Tca8113 cells can secrete a large amount of vesicle-like structures, which are identified as exosomes by the presence of the surface markers, Hsp-70 and Alix. The protein profile of the two products are almost the same, however the particle size distribution of the exosomes extracted with density gradient centrifugation are more limited, between 40-120 $\mathrm{nm}$, and these have a higher protein concentration. The results indicate that Tca8113 cells can secrete exosomes in vitro, and the density gradient separation methods for purifying exosomes is improved, which is helpful for future research and application of exosomes.
\end{abstract}

\section{Introduction}

Exosomes are bioactive vesicles derived from the endosomal membrane system of the cell, followed by secretion into the surrounding body fluids, with diameters ranging from $40-100 \mathrm{~nm}$ and a density from $1.13-1.19 \mathrm{~g} / \mathrm{ml}$. Exosomes were

Correspondence to: Professor Longjiang Li, State Key Laboratory of Oral Diseases or Department of Head and Neck Cancer Surgery, West China School of Stomatology, Sichuan University, No. 14, 3rd Section of Ren Min Nan Road, Chengdu, Sichuan 610041, P.R. China

E-mail: muzili63@163.com

Key words: tongue squamous cell carcinoma, exosome, ultracentrifugation, density gradient separation officially named by Johnstone et al in 1987, who found that microvesicles can assist maturing erythrocytes to eliminate the transferring receptors as well as other dumped proteins (1). However, numerous studies have identified that exosomes can be produced by various types of cells, including different types of epithelial and nerve cells (2), and have even been detected in various body fluids (3-5). Exosomes are saucer-like vesicles under electron microscopy, which contain special proteins, lipids, RNA and micro-RNAs $(6,7)$. They can be identified by Alix, Tsg101, heat shock protein 70 (Hsp70), and cluster of differentiation 63 (CD63), CD81 and CD9, and they play different roles in various pathological conditions. Thus far, in addition to participating in metabolizing products, it has been demonstrated that exosomes possess a number of biological functions, including immune regulation, communication of cells, matrix remodeling, signaling pathways activating through transferring growth factor or receptor, intercellular exchanging of oncoprotein and oncogene, and induction of angiogenesis and regulation of the treatment reaction. In particular, their significant role in intercellular communication has gained more and more attention in studies $(1,8-11)$. Currently, there are three main methods for extraction of exosomes: i) Classic ultracentrifugation (12); ii) density gradient centrifugation, which is widely used at present (13); and iii) the technology of immunomagnetic capture (14-16). Due to the high cost and lack of specific antigens, the certain advantages of magnetic bead technology have resulted in its limited application. At present, the other two methods have a lack of a systematic comparison. The aim of the present study was to demonstrate that the Tca8113 human tongue squamous cell carcinoma cell line can secrete exosomes, and to perform an extensive evaluation of the methods widely used for extracting the exosomes, including density gradient centrifugation and ultracentrifugation.

\section{Materials and methods}

Cell culture and collection of required culture medium. The human tongue squamous cell carcinoma cell line, Tca8113, was obtained from the Cell Institute, Chinese Academy of Sciences (Shanghai, China) and cultured in Dulbecco's 
modified Eagle's medium (DMEM; Hyclone Laboratories, Inc., Logan, UT, USA) containing 10\% fetal bovine serum (FBS; Gibco-BRL, Carlsbad, CA, USA) with $5 \% \mathrm{CO}_{2}$ at $37^{\circ} \mathrm{C}$, and cells were passaged when they were $90-100 \%$ confluent. Tca8113 cells were washed twice with $10 \mathrm{ml}$ phosphate-buffered saline (PBS) and cultured for $48 \mathrm{~h}$ in $5 \mathrm{ml}$ DMEM media with $10 \%$ FBS, which was previously centrifuged at $100,000 \mathrm{x} \mathrm{g}$ for $70 \mathrm{~min}$ to eliminate bovine-derived exosomes. Subsequently, $50 \mathrm{ml}$ culture medium (CM) was collected and stored at $-20^{\circ} \mathrm{C}$ for later use.

Ultracentrifugation exosome (UC-Exo) isolation. Exosomes were isolated from the required cell culture supernatant as described previously (12). Briefly, the cell culture supernatant was centrifuged $(300 \mathrm{x} \mathrm{g}$ for $10 \mathrm{~min} ; 2,000 \mathrm{x} \mathrm{g}$ for $20 \mathrm{~min}$ to eliminate dead cells; and $10,000 \mathrm{x} \mathrm{g}$ for $30 \mathrm{~min}$ to remove debris) and then pelleted by ultracentrifugation at $100,000 \mathrm{x} \mathrm{g}$ for $70 \mathrm{~min}$ at $4^{\circ} \mathrm{C}$. The pellet was resuspended in $1 \mathrm{ml}$ PBS and re-centrifuged (100,000 x g, $70 \mathrm{~min})$, as aforementioned. The products (UC-Exo) were resuspended in $200 \mu \mathrm{l}$ PBS and passed through $0.22-\mu \mathrm{m}$ microcentrifuge filters (Sigma-Aldrich, St. Louis, MO, USA) prior to being stored at $-80^{\circ} \mathrm{C}$.

Density gradientexosome (DG-Exo) isolation.Exosomes were isolated using a method described by Lamparski et al (17). Briefly, the collected CM was centrifuged at $2000 \mathrm{x} g$ for $30 \mathrm{~min}$ to remove cellular debris. The processed supernatant was concentrated by centrifugation for $50 \mathrm{~min}$ at $1,000 \mathrm{x} \mathrm{g}$ in a $100 \mathrm{kDa}$ molecular weight cut-off hollow-fibre membrane (Millipore, Bedford, MA, USA). The concentrated products were collected and added to an ultracentrifuge tube with a $30 \%$ sucrose $/ \mathrm{D}_{2} \mathrm{O}$ cushion (density, $1.210 \mathrm{~g} / \mathrm{cm}^{3}$ ) at the bottom of the tube, followed by ultracentrifugation (Sorvall Ultra Pro 80; Kendro Laboratory Products Ltd., Newtown, CT, USA) in a Surespin 630 swinging bucket (Thermo Fisher Scientific, Rockford, IL, USA) at $100,000 \times \mathrm{g}$ at $4^{\circ} \mathrm{C}$ for $70 \mathrm{~min}$. Subsequently, the cushion was collected, followed by being washed and concentrated twice with PBS by centrifuging for $50 \mathrm{~min}$ at $1,000 \mathrm{x} \mathrm{g}$ in the aforementioned capsule. The following steps were consistent with the former method.

Analysis of the laser particle size. The analysis of the particle size was carried out and repeated three times with a laser diffraction instrument (Malvern Zetasizer Nano ZS90; Malvern Instruments Ltd., Malvern, UK). A 30- $\mu$ l aliquot of exosomes isolated respectively from the two types of methods was diluted with PBS to $1 \mathrm{ml}$ and transferred to the specific tube subsequent to repeatedly blowing. The particle size was measured every $0.5 \mathrm{~min}$ for the duration of the test and the results were automatically recorded.

Transmission electron microscopy (TEM). The process of observation through TEM were performed as previously described (16) with slight alterations. Briefly, a $20 \mu \mathrm{l}$ aliquot of exosome preparations were placed onto formvar-coated 200-mesh copper grids (ProSciTech, Queensland, Australia) for $1 \mathrm{~min}$ at room temperature and allowed to dry through filter paper. The grids were subsequently washed twice with water for $5 \mathrm{~min}$ and stained with $20 \mathrm{~g} / \mathrm{l}$ uranyl acetate in water (ProSciTech) for $1 \mathrm{~min}$. The grids were examined at an acceleration voltage of $100 \mathrm{kV}$ using a JEOL JEM-2100 TEM (JEOL USA, Inc., Peabody, MA, USA).

Protein extraction and concentration determination. As a result of the pre-experiment, the highest concentration of protein appeared in the certain instances when $100 \mu \mathrm{l}$ lysis buffer was added to the same volume of samples. According to the total protein extraction kit instructions (Bi Yuntian Biological Technology Institution, Shanghai, China), $100 \mu 1$ lysis buffer was added to the same volume of samples on ice, followed by shaking wildly on the horizontal shaking platform for $15 \mathrm{~min}$ and centrifuging at $15,558 \mathrm{x} \mathrm{g}$ at $4^{\circ} \mathrm{C}$ for another $15 \mathrm{~min}$. Subsequently, the supernatant was obtained. Finally, a $20-\mu 1$ aliquot of the supernatants was used for the determination of the protein concentration with the bicinchoninic acid (BCA) Protein Assay kit (Bi Yuntian Biological Technology Institution).

SDS-page gel electrophoresis with Coomassie brilliant blue staining and western blotting. Total cellular proteins were loaded and run on $10 \%$ SDS gels (Beyotime Institute of Biotechnology, Shanghai, China) and then either transferred onto polyvinylidene fluoride membranes (Bio-Rad, Hercules, CA, USA) or stained with Coomassie brilliant blue (Beyotime Institute of Biotechnology). The membranes were blocked in 5\% (w/v) skimmed milk in Tris-buffered saline with Tween 20 and incubated at $4^{\circ} \mathrm{C}$ with primary antibodies against monoclonal mouse anti-human Alix (1:1,000; Santa Cruz Biotechnology, Inc., Santa Cruz, CA, USA) or Hsp70 (1:1,000; Santa Cruz Biotechnology, Inc.) overnight. The polyclonal rabbit anti-goat IgG-horseradish peroxidase secondary antibodies (Wuhan Boster Biological Technology, Ltd., Wuhan, China) were incubated at room temperature for $1 \mathrm{~h}$ and the membranes were visualized by the Amersham ECL Select detection system (Amersham Pharmacia Biotech, Little Chalfont, UK).

Statistical analysis. All data were performed using SPSS 13.0 (SPSS, Inc., Chicago, IL, USA) statistical software. A paired t-test was used to compare the protein concentration between the two methods. $\mathrm{P}<0.05$ was considered to indicate a statistically significant difference.

\section{Results}

Particle size distribution of the exosomes. The particle size of the exosomes isolated respectively from the two types of method were measured by a laser diffraction instrument. The results revealed that the range of the particle size of UC-Exo (Fig. 1A) was wider than that of DG-Exo (Fig. 1B), even $>200 \mathrm{~nm}$, whilst the diameters of DG-Exo were mostly in a uniform range from 30-120 $\mathrm{nm}$. The values of the polydispersity index were 0.387 and 0.481 for UC-Exo and DG-Exo, respectively, and were within the normal range.

Morphology observation of the exosomes. Morphological analysis of the UC-Exo and DG-Exo samples using TEM revealed the same results that the vesicles were comprising round-shaped 30-150-nm diameter vesicles, which is consistent with the aforementioned exosomes (Fig. 2), while the 
A

\begin{tabular}{|c|c|c|c|c|c|}
\hline & & & Size (d.nm): & $\%$ Intensity & Width (d.nm): \\
\hline Z-Average (d.nm): & 76.46 & Peak 1: & 169.3 & 60.6 & 53.51 \\
\hline Pdl: & 0.387 & Peak 2: & 18.45 & 38.3 & 4.953 \\
\hline Intercept: & 0.889 & Peak 3: & 5560 & 1.1 & $6.104 e-5$ \\
\hline
\end{tabular}

Result quality : Refer to quality report

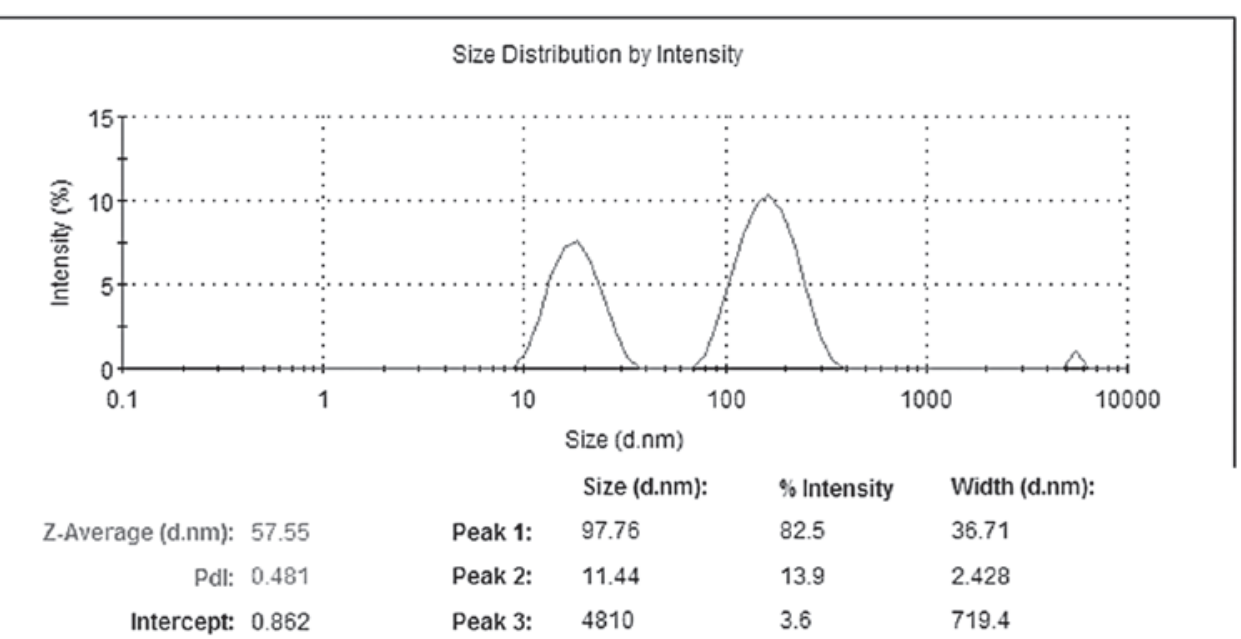

Result quality : Refer to quality report

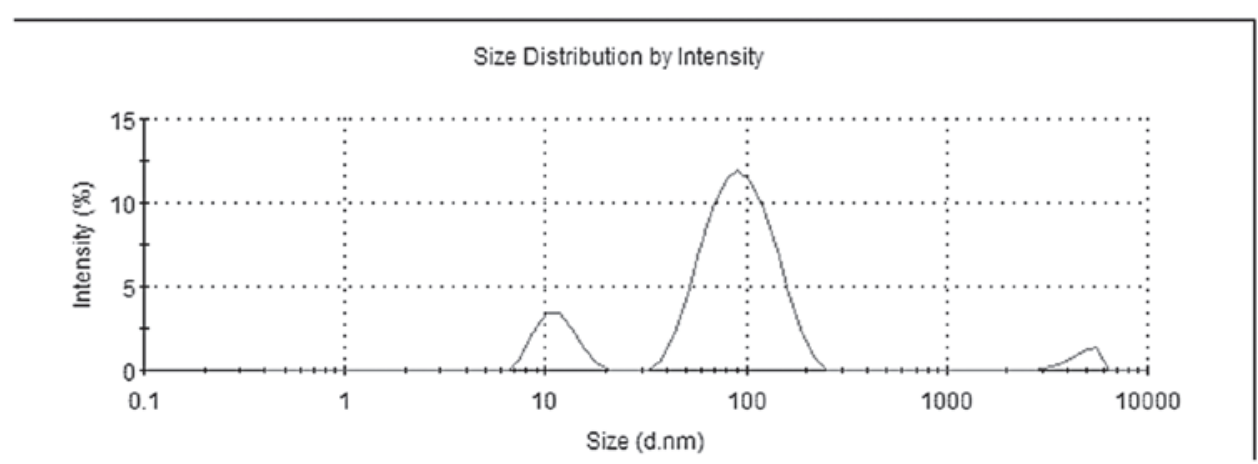

Figure 1. Particle size of exosomes isolated respectively from two kinds of methods were measured by a laser diffraction instrument. (A) The UC-Exo were widely distributed, $>200 \mathrm{~nm}$. (B) The diameter of DG-Exo mostly ranged uniformly from 30 to $120 \mathrm{~nm}$. UC-Exo, ultracentrifugation exosome; DC-Exo, density gradient-Exo.
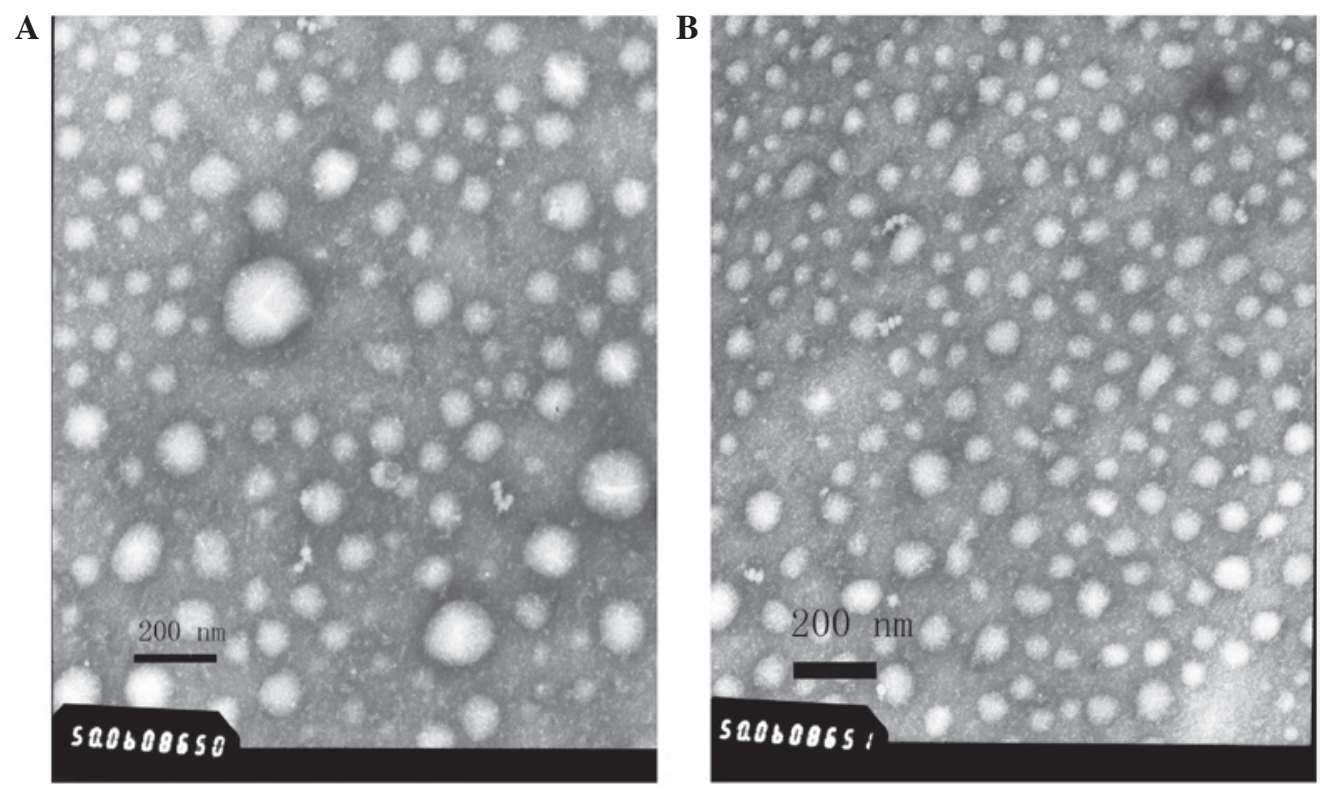

Figure 2. (A) UC-Exos and (B) DG-Exos were characterized by electron microscopy. The results showed nano-sized vesicles of a 30-150-nm diameter, with a 'round-shaped' morphology. Exosomes from each method were negatively stained with uranyl acetate and viewed by electron microscopy. The scale bar represents $200 \mathrm{~nm}$ (magnification, x50,000). UC-Exo, ultracentrifugation exosome; DC-Exo, density gradient-Exo. 
Table I. Determination of protein concentration of exosomes purified by two different methods.

\begin{tabular}{rcc}
\hline Sample & $\begin{array}{c}\text { UC-Exo } \\
\text { concentration, } \mu \mathrm{g} / \mathrm{ml}\end{array}$ & $\begin{array}{c}\text { DG-Exo } \\
\text { concentration, } \mu \mathrm{g} / \mathrm{ml}\end{array}$ \\
\hline 1 & 174.957 & 380.910 \\
2 & 132.555 & 467.076 \\
3 & 75.361 & 364.452 \\
4 & 179.888 & 288.936 \\
5 & 64.325 & 410.922 \\
6 & 103.051 & 471.916 \\
7 & 135.000 & 535.814 \\
8 & 165.013 & 410.922 \\
9 & 163.076 & 411.892 \\
10 & 132.096 & 427.382 \\
11 & 133.064 & 329.598 \\
12 & 252.146 & 327.622 \\
\hline
\end{tabular}

UC-Exo, ultracentrifugation exosome; DC-Exo, density gradient-Exo.

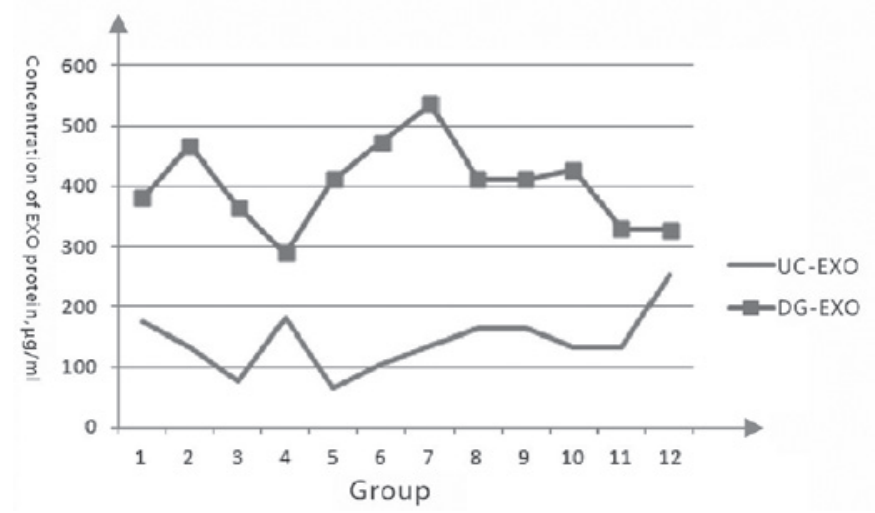

Figure 3. Results of a line graph derived from Table I. A paired t-test, $\mathrm{P}<0.05$, implies that the difference was statistically significant.

differences with the traditional cup-shape may be associated with the preparation of the samples. However, certain UC-Exo (Fig. 2A) samples contained vesicles that were $>200 \mathrm{~nm}$ in diameter, which could even be suspected to be a class of apoptosis body, while the DG-Exo were well-distributed (Fig. 2B).

Determination of the protein concentration of the exosomes. The determination of the total protein concentration of the UC-Exo and DG-Exo samples were compared using the BCA method. The measurement included 12 separate samples from UC-Exo and DG-Exo (Table I) and the correlation coefficient was 0.99. Fig. 3 shows the curve comparison chart generated by Table I, with a paired t-test, $\mathrm{P}<0.05$, which indicated that the difference had a statistical significance.

Protein composition of the exosomes. The proteins were separated by SDS-PAGE gel electrophoresis and stained by Coomassie brilliant blue. The results showed that the bands of the two samples were almost the same and were mainly

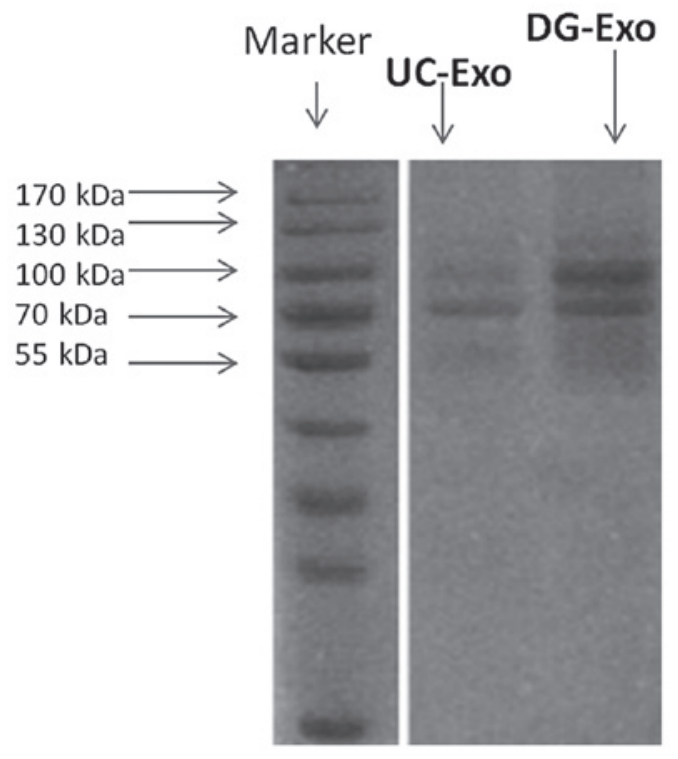

Figure 4. Protein composition of exosomes. The first longitudinal stripe on the right side was the marker, which was the indicator of molecular weight of the protein, while the other two longitudinal stripes represented the distribution of protein extracted from UC-Exo (left) and DG-Exo (right), respectively. The bands of the groups were similar, which contained the molecular weights of 55, 70, 100 and $130 \mathrm{kDa}$, while $170 \mathrm{kDa}$ was not obvious. UC-Exo, ultracentrifugation exosome; DC-Exo, density gradient-Exo.

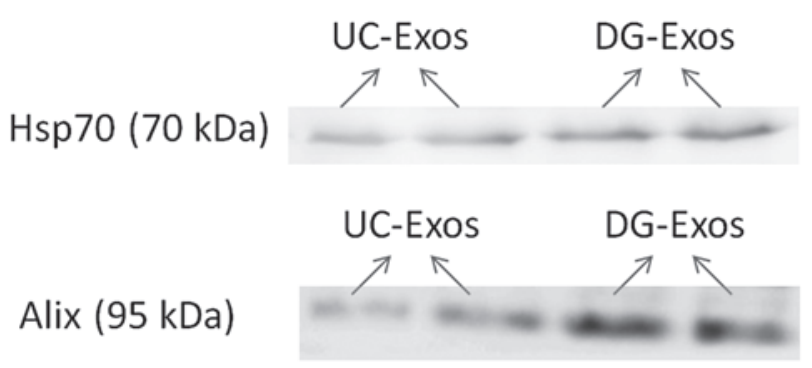

Figure 5. UC-Exos and DG-Exos were characterized by western blotting. For western blotting, each exosome preparation $(20 \mu 1)$ was separated by 1D-SDS-PAGE, followed by being transferred onto polyvinylidene fluoride membranes, and probed with exosome markers Alix and Hsp70. UC-Exo, ultracentrifugation exosome; DC-Exo, density gradient-Exo; Hsp70, heat shock protein 70 .

distributed between 40-130 kDa with obvious separated strips (Fig. 4).

Identification of the molecular markers. The molecular phenotype of the exosomes was typical of exosomes from other sources. In the present study, it was found that Hsp70 and Alix, the protein markers of exosomes, were detected similarly in the purified exosomes with various methods (Fig. 5).

\section{Discussion}

Exosomes may participate in cell communication by delivering proteins, RNA and miRNA (18-20) and has the ability to induce or suppress the immune system (21). Exosomes are capable of promoting angiogenesis, remodelling the microenvironment and promoting tumor growth $(22,23)$. Their use in diagnosis and treatment have been confirmed $(3,4,24)$. 
However, the current area of study offering the most promise lies with isolating and extracting high quality exosomes.

The significant reference standard to judge exosomes purification methods is based on the ability to remove other membranous particles and concentrate protein. Although ultracentrifugation is the most widely used method for exosomes isolation, it is limiting in these respects, so a more specific method is required. In the present study, two strategies were compared for purifying the human tongue cancer cell line Tca8113-derived exosomes; ultracentrifugation and density gradient separation. The efficacy of the two strategies was judged by TEM, particle size distribution and cursory proteome profiling of the enrichment of typical exosomal markers, including Alix and Hsp70, which was confirmed by western blot analysis and protein concentration.

Experimental results show that the exosomes extracted from the two methods, comprising round-shaped 30-150-nm diameter vesicles, are consistent with exosomes reported previously $(16,25)$. However, the coexistence of the larger vesicles with UC-Exo is unknown and presumably not due to the vesicles being slightly clumped together or due to other mixed impurities. Another study has noted that steps, including freezing and thawing, or multiple centrifugal steps do not affect exosome size and shape (17). Western blot analysis revealed the presence of the exosome markers Alix and Hsp70 in both methods, which showed that both of the methods can extract the exosomes. In addition, the results of laser particle size measurement revealed that UC-Exo had a wider range of diameter distribution and a larger proportion of vesicles with diameters $>200 \mathrm{~nm}$, while the DG-Exo was more uniform and the majority of diameters were distributed between 30-150 nm with an average of $87.3 \mathrm{~nm}$. This shows that the latter samples have a higher purity. Through SDS-PAGE gel electrophoresis and Coomassie brilliant blue staining, the bands of the two samples were found to be almost the same and mainly distributed between 40-130 kDa with obvious separated strips, while the $170 \mathrm{kDa}$ was not obvious. However, by comparing the two methods with protein concentration and western blot semi-quantitative analysis, it was found that the total protein concentration of DG-Exo was higher and had a higher proportion of target protein, which indicated that density gradient centrifugation has a higher extraction efficiency. Additionally, the density gradient centrifugation used a $30 \%$ sucrose/heavy water cushion, which has a density distribution range that is consistent with exosomes and is a type of variable purification, and has been used widely to purify exosomes (26). The repeated purification with ultrafiltration also produces samples with a higher purity and improved quality. Therefore, density gradient centrifugation is more comprehensive and more efficient than ultracentrifugation in the extraction of exosomes. However, there are specific problems attached, including the higher cost, the higher requirements of equipment and technical ability, the time required is longer and the separation and extraction of heavy water at a high-quality is difficult. In addition, future studies could be conducted to identify whether the cell damage from heavy water can be simply eliminated by repeated dilution of the purification and whether this will effect the subsequent experiment, whether the material existing in the extraction of the sample whose diameter is $>200 \mathrm{~nm}$ can be further purified or excluded and whether one or more specific markers and extraction methods for exosomes will be a problem that requires solving. In conclusion, both preparations contained vesicles with sizes of 30-150 nm and expression of the exosome markers, Alix and HSP70. However, density gradient separation was considered to be the efficient method to isolate exosomes, as it was able to enrich exosome markers, and exosome-associated proteins by at least two-fold more than the other methods studied. Protein, lipid, mRNA and microRNA analyses of highly-purified vesicles will lead to significant advances in exosome characterization, and facilitate a deeper understanding of their biological functions.

\section{Acknowledgements}

This study was supported by the National Natural Science Foundation (grant no. 81172578).

\section{References}

1. Johnstone RM, Adam M, Hammond JR, et al: Vesicle formation during reticulocyte maturation. Association of plasma membrane activities with released vesicles (exosomes). J Biol Chem 262: 9412-9420, 1987

2. Cocucci E, Racchetti G and Meldolesi J: Shedding microvesicles: artefacts no more. Trends Cell Biol 19: 43-51, 2009.

3. Gonzales PA, Pisitkun T, Hoffert JD, Tchapyjnikov D, Star RA, Kleta R, et al: Large-scale proteomics and phosphoproteomics of urinary exosomes. J Am Soc Nephrol 20: 363-379, 2009.

4. Nilsson J, Skog J, Nordstrand A, Baranov V, Mincheva-Nilsson L, Breakefield XO and Widmark A: Prostate cancer-derived urine exosomes: a novel approach to biomarkers for prostate cancer. $\mathrm{Br} \mathrm{J}$ Cancer 100: 1603-1607, 2009.

5. Michael A, Bajracharya SD, Yuen PS, Zhou H, Star RA, Illei GG and Alevizos I: Exosomes from human saliva as a source of microRNA biomarkers. Oral Dis 16: 34-38, 2010.

6. Skog J, Würdinger T, van Rijn S, et al: Glioblastoma microvesicles transport RNA and proteins that promote tumour growth and provide diagnostic biomarkers. Nat Cell Biol 10: 1470-1476, 2008.

7. Hong BS, Cho JH, Kim H, et al: Colorectal cancer cell-derived microvesicles are enriched in cell cycle-related mRNAs that promote proliferation of endothelial cells. BMC Genomics 10: 556, 2009.

8. Zhang HG and Grizzle WE: Exosomes and cancer: a newly described pathway of immune suppression. Clin Cancer Res 17: 959-964, 2011

9. Keller S, Sanderson MP, Stoeck A and Altevogt P: Exosomes: from biogenesis and secretion to biological function. Immunol Lett 107: 102-108, 2006.

10. Simons M and Raposo G: Exosomes - vesicular carriers for intercellular communication. Curr Opp Cell Biol 21: 575-581, 2009.

11. Mathivanan S, Ji H and Simpson RJ: Exosomes: extracellular organelles important in intercellular communication. J Proteomics 73: 1907-1920, 2010.

12. Raposo G, Nijman HW, Stoorvogel W, Liejendekker R, Harding CV, Melief CJ and Geuze HJ: B lymphocytes secrete antigen-presenting vesicles. J Exp Med 183: 1161-1172, 1996.

13. Ostrowski M, Carmo NB, Krumeich S, et al: Rab27a and Rab27b control different steps of the exosome secretion pathway. Nat Cell Biol 12: 19-30, 2010.

14. Rabinowits G, Gerçel-Taylor C, Day JM, Taylor DD and Kloecker GH: Exosomal microRNA: a diagnostic marker for lung cancer. Clin Lung Cancer 10: 42-46, 2009.

15. Taylor DD and Gercel-Taylor C: MicroRNA signatures of tumor-derived exosomes as diagnostic biomarkers of ovarian cancer. Gynecol Oncol 110: 13-21, 2008.

16. Mathivanan S, Lim JW, Tauro BJ, Ji H, Moritz RL and Simpson RJ: Proteomics analysis of A33 immunoaffinity-purified exosomes released from the human colon tumor cell line LIM1215 reveals a tissue-specific protein signature. Mol Cell Proteomics 9: 197-208, 2010.

17. Lamparski HG, Metha-Damani A, Yao JY, Patel S, Hsu DH, Ruegg C and Le Pecq JB: Production and characterization of clinical grade exosomes derived from dendritic cells. J Immunol Methods 270: 211-226, 2002. 
18. Chairoungdua A, Smith DL, Pochard P, Hull M and Caplan MJ: Exosome release of beta-catenin: a novel mechanism that antagonizes Wnt signalling. J Cell Biol 190: 1079-1091,2010.

19. Verweij FJ, van Eijndhoven MA, Hopmans ES, et al: LMP1 association with CD63 in endosomes and secretion via exosomes limits constitutive NF-kappaB activation. EMBO J 30: 2115-2129, 2011.

20. Ohshima K, Inoue K, Fujiwara A, et al: Let-7 microRNA family is selectively secreted into the extracellular environment via exosomes in a metastatic gastric cancer cell line. PloS One 5: e13247, 2010.

21. Clayton A, Al-Taei S, Webber J, Mason MD and Tabi Z: Cancer exosomes express CD39 and CD73, which suppress T cells through adenosine production. J Immunol 187: 676-683, 2011.

22. Xiang X, Poliakov A, Liu C, et al: Induction of myeloid-derived suppressor cells by tumor exosomes. Int J Cancer 124: 2621-2633, 2009
23. CastellanaD,ZobairiF,MartinezMC,etal:Membrane microvesicles as actors in the establishment of a favorable prostatic tumoral niche: a role for activated fibroblasts and CX3CL1-CX3CR1 axis. Cancer Res 69: 785-793, 2009.

24. Xiao WH, Sanren GW, Zhu JH, et al: Effect of 5-aza-2'-deoxycytidine on immune-associated proteins in exosomes from hepatoma. World J Gastroenterol 16: 2371-2377, 2010.

25. Regev-Rudzki N, Wilson DW, Carvalho TG, et al: Cell-cell communication between malaria-infected red blood cells via exosome-like vesicles. Cell 153: 1120-1133, 2013.

26. Lamparski HG, Metha-Damani A, Yao JY, Patel S, Hsu DU, Ruegg $\mathrm{C}$ and LePecq JB: Production and characterization of clinical grade exosomes derived from dendritic cells. J Immunol Methods 270: 211-226, 2002. 